\title{
BMJ Open Evaluating the association of preoperative parecoxib with acute pain trajectories after video-assisted thoracoscopic surgery: a single-centre cohort study in Taiwan
}

\author{
Yu-Hsiang Ling, ${ }^{1,2}$ Ying-Hsuan Tai, ${ }^{2,3,4,5}$ Hsiang-Ling Wu, ${ }^{2,3}$ Wei-Lun Fu, ${ }^{3}$ \\ Mei-Yung Tsou, ${ }^{2,3}$ Kuang-Yi Chang (iD) 2,3
}

To cite: Ling Y-H, Tai Y-H, Wu H-L, et al. Evaluating the association of preoperative parecoxib with acute pain trajectories after videoassisted thoracoscopic surgery: a single-centre cohort study in Taiwan. BMJ Open 2021;11:e038985. doi:10.1136/ bmjopen-2020-038985

- Prepublication history and additional material for this paper is available online. To view these files, please visit the journal online (http://dx.doi.org/10. 1136/bmjopen-2020-038985).

Received 31 March 2020 Revised 27 October 2020 Accepted 21 January 2021

Check for updates

(C) Author(s) (or their employer(s)) 2021. Re-use permitted under CC BY-NC. No commercial re-use. See rights and permissions. Published by BMJ.

For numbered affiliations see end of article.

Correspondence to Dr Kuang-Yi Chang; kychang@vghtpe.gov.tw

\section{ABSTRACT}

Objective The efficacy of parecoxib as pre-emptive analgesia still remains controversial. This study aimed to investigate how pre-emptive analgesia with parecoxib affected postoperative pain trajectories over time in patients undergoing thoracic surgery.

Design Retrospective cohort study.

Setting A single medical centre in Taiwan.

Participants We collected 515 patients undergoing videoassisted thoracoscopic surgery at a tertiary medical centre between September 2016 and August 2017.

Interventions Pre-emptive parecoxib before surgery.

Primary and secondary outcome measures Daily numeric rating pain scores in the first postoperative week. Results A total of 196 (38.1\%) of the recruited patients received parecoxib preoperatively. The latent curve analysis revealed that woman, higher body weight and postoperative use of parecoxib were associated with increased baseline level of pain scores over time $(p=0.035$, 0.005 and 0.048 , respectively) but epidural analgesia and preoperative use of parecoxib were inclined to decrease it (both $p<0.001$ ). Regarding the decreasing trends of changes in daily pain scores, older age and epidural analgesia tended to steepen the slope $(p=0.014$ and $<0.001$, respectively). Preoperative use of parecoxib were also related to decreased frequency of rescue morphine medication ( $\mathrm{HR}=0.4 ; 95 \% \mathrm{Cl} 0.25$ to 0.65 ).

Conclusions Pre-emptive analgesia with parecoxib was associated with decreased baseline pain scores but had no connection with pain decreasing trends over time. Latent curve analysis provided insights into the dynamic relationships among the analgesic modalities, patient characteristics and postoperative pain trajectories.

\section{INTRODUCTION}

Pre-emptive analgesia has been introduced to relieve postoperative pain for decades. ${ }^{1}$ The concept of pre-emptive analgesia is based on the central sensitisation caused by peripheral nociceptor hypersensitivity. ${ }^{2}$ Inflammatory mediators are released locally by the tissue injured from a surgical incision, causing
Strengths and limitations of this study

- Latent curve analysis revealed the complex relationship between dynamic changes of postoperative pain scores over time, various types of analgesic modalities and patient characteristics in the clinical setting of video-assisted thoracoscopic surgery.

- It is noted that parecoxib mainly exerted its preemptive analgesic effects on the baseline value rather than decreasing rate of postoperative pain scores.

- It is difficult to assess potential confounding effects from unmeasured variables, such as preoperative pain and psychosocial factors.

- The numerical rating score is a direct but relatively subjective indicator in the measurement of pain intensity.

nociceptor hypersensitivity and hyperalgesia of the injured tissue. The signal of pain is transduced to dorsal horn through A-delta and $\mathrm{C}$ fibrers and then transduced to thalamus and cortex through the spinothalamic tract, thereby triggering central sensitisation. ${ }^{2}$ The preoperative administration of analgesics can attenuate the release of peripheral inflammatory mediators and relieve postoperative pain. Efficacy of pre-emptive analgesia has been confirmed by many randomised controlled trials in various types of surgery. ${ }^{3}$ Parecoxib, a COX-2 selective inhibitor, is a water-soluble and injectable prodrug of valdecoxib. Parecoxib is used mostly for short-term perioperative pain control, and a recent clinical trial reported that pre-emptive administration of parecoxib can significantly reduce the risk of ipsilateral shoulder pain after thoracotomy. ${ }^{4}$ Although the efficacy of pre-emptive analgesia with intravenous parecoxib has been well described, ${ }^{4-9}$ its correlation with the 
postoperative pain trajectories over time has not been established.

In pain medicine, latent curve analysis provides the information about postoperative numeric pain scores, analgesics demand and their patterns of change over time, under different interventions or patient characteristics. ${ }^{11}$ Accordingly, we applied latent curve model to analyse the variation in daily pain score measurements in patients undergoing video-assisted thoracoscopic surgery, with or without pre-emptive administration of parecoxib. The study aimed to investigate how pre-emptive use of parecoxib affected postoperative pain trajectories over time. We hypothesised pre-emptive parecoxib was not only associated with lower baseline of postoperative pain trajectories but also connected to the pattern of pain resolution over time. In addition, we also postulated that pre-emptive parecoxib was inclined to reduce both the probability and frequency of rescue medication. Furthermore, a prediction model using latent curve analysis for quantitively describing postoperative pain resolution was constructed. Other influential factors of postoperative pain were also assessed to provide a more comprehensive view of dynamic change in postoperative pain trajectories.

\section{MATERIALS AND METHODS}

\section{Setting and patient selection}

Patients undergoing video-assisted thoracoscopic surgery for resection of pulmonary neoplasms at this tertiary medical centre between September 2016 and August 2017 were identified from the institutional electronic medical record system. Patients were excluded from the analysis if they had critical missing data about demographics, analgesic management or $<2$ postoperative pain assessments during the hospital stay.

\section{Patient and public involvement}

No patient involved.

\section{Anaesthesia and analgesia management}

All patients undergoing video-assisted thoracoscopic surgery were given balanced general anaesthesia at this centre, including fentanyl $1-2 \mu \mathrm{g} \mathrm{kg} \mathrm{kg}^{-1}$ and propofol $1-2 \mathrm{mg} \mathrm{kg}^{-1}$ for induction, and neuromuscular blocking drugs to facilitate tracheal intubation with rocuronium $0.8 \mathrm{mg} \mathrm{kg}^{-1}$ or cisatracurium $0.2 \mathrm{mg} \mathrm{kg}^{-1}$. Anaesthesia was maintained with sevoflurane $2-3$ vol\% or desflurane $6-8$ vol\% in a mixture of oxygen and air.

Patients were offered four options of analgesic modalities, including patient-controlled epidural analgesia (PCEA), intravenous patient-controlled analgesia (IVPCA), intravenous administration of parecoxib and intravenous as-needed analgesia. If PCEA was selected, epidural catheters were typically placed at a middle thoracic spine (eg, T6-T8) and assessed its function with a test dose of local anaesthetic preoperatively. Epidural analgesia was started intraoperatively with a combination of local anaesthetic (bupivacaine $0.25 \%$ or $0.5 \%$ ) and $5 \mu \mathrm{g} \mathrm{mL} \mathrm{L}^{-1}$ fentanyl, and continued postoperatively for 48-72 hours. Patients receiving IVPCA was administered via an ambulatory infusion pump (Gemstar Yellow, Hospira, Illinois, USA) programmed to deliver morphine at a demand dose of $1 \mathrm{mg}$ with a lockout time of $6 \mathrm{~min}$ for 48-72 hours after surgery. ${ }^{12}$ PCEA and IVPCA were assessed daily for proper function by the acute pain service team. Patients receiving parecoxib for postoperative pain control were treated with two regimens, either intravenous administration of parecoxib $40 \mathrm{mg}$ intravenously 30 min before operation or immediately after operation, followed by parecoxib 20-40 mg every 12 hours for 3 days. Those not receiving the preceding analgesic treatment were given intravenous as-needed analgesia with narcotics or non-steroidal anti-inflammatory drugs. Intravenous or intramuscular morphine $5 \mathrm{mg}$ was administered as rescue medication and the total number of times of rescue morphine use during the first three postoperative days (PODs) were collected as well.

\section{Data retrieval}

Patient characteristics and the maximum of daily pain scores using an 11-point numeric rating scale (NRS) with response options from 'no pain' to 'the worst pain' during the first postoperative week were retrieved from the electronic medical record system and served as the study endpoints in the following latent curve analysis. Medical records of the recruited patients were extracted by a specialist anaesthesiologist who were not involved in statistical analysis. Random samples of the extracted data were thoroughly checked by the authors to ensure the quality of data.

\section{Statistical analysis}

All study subjects were classified into two groups based on whether parecoxib was used preoperatively or not. Patient characteristics and maximal daily pain scores during the first postoperative week were expressed as mean $\pm \mathrm{SD}$, count with percentage or median with IQR as appropriate. The comparisons of patient characteristics between the two groups were performed using $\chi^{2}$ tests for categorical variables and either t-tests or Wilcoxon rank-sum tests for continuous variables. Linear mixed models were used to compare maximal daily pain scores in distinct PODs between the two groups. Latent curve analysis was performed to model the change of daily maximal pain scores over time and evaluate how analgesic modalities and patient characteristics affected the trajectory of pain scores. Three latent curve models, the basic, single predictor and multiple predictors models, were applied to explore the transition of daily pain scores over time. ${ }^{910}$ The basic model was applied to estimate the baseline intercept and slope parameters, and then the single predictor model was used to evaluate the effect of covariates in this study on the intercept and slope parameters, while the backward model selection strategy was used to identify independent explanatory factors of the intercept and slope parameters in the multiple predictors 
model analysis. Prediction models based on latent curve analysis were also developed. The details of statistical technique on latent curve models refer to the previous literature. ${ }^{1314}$ Root mean square error of approximation (RMSEA) and comparative fit index (CFI) were used to evaluate model fit. The values of RMSEA $<0.1$ and CFI $>0.9$ implied acceptable fit to data. ${ }^{15} 16$ Hurdle Poisson regression model was applied to analyse probability and frequency of rescue morphine use and to accomodate the large number of zeros and positively skewed nonzero counts in usage data. ${ }^{17}$ Hurdle Poisson regression model includes two components: the binary part which determines whether the responses overcome the hurdle or not, and the truncated Poisson part for modelling the responses above the hurdle. Predictors included in the model were age, sex, body weight, preoperative use of parecoxib, postoperative use of parecoxib, IVPCA and PCEA. All latent curve analyses were implemented using AMOS V.18.0 (SPSS, Chicago, Illinois, USA). Other statistical analyses were conducted with SAS V.9.4 software (SAS Institute).

\section{RESULTS}

A total of 515 patients were included in the analysis and 196 of these patients (38.1\%) had pre-emptive analgesia with parecoxib. The average pain scores of all study subjects were 2.7 on the POD 1 , peaked at 2.8 on the POD 2, and then decreased gradually to 1.9 on the POD 7. Table 1 presents the comparisons of patient characteristics and postoperative pain scores between preoperative parecoxib users and their counterparts. Note that most patients among the preoperative parecoxib users continued to take this medication postoperatively $(92.3 \%)$. Besides, on average, patients of the preoperative parecoxib group tended to have significantly lower pain scores in 6 of the 7 PODs despite the minimal difference but had a higher likelihood of using rescue morphine $(70.9 \%$ vs $26.3 \%, \mathrm{p}<0.001)$ than non-users. Patients in the preoperative parecoxib group also had shorter length of hospital stay than non-users $(\mathrm{p}<0.001)$.

The estimated factor loading of slope parameters from the POD 2-6 ranged between -0.04 and 0.85 (figure 1). For example, the difference in the average pain scores between the POD 1 and 3 was only $33 \%$ of that between the POD 1 and $7(\mathrm{p}=0.44)$. Note that the slope parameter of the POD 2 was not significant, which means there was no significant difference in the average pain score between the POD 1 and 2. The estimated values of intercept and slope parameters of the basic latent curve model were 2.76 and -1 , respectively. Accordingly, the estimated daily pain score during the first postoperative week can be calculated as follows:

NRS pain score on POD 1: $2.74+0 \times(-1)=2.74$; POD 2: $2.74-0.04 \times(-1)=2.78$; POD 3: $2.74+0.33 \times(-1)=2.41$; POD 4: $2.74+0.54 \times(-1)=2.20 ; \quad$ POD 5: $2.74+0.72 \times(-1)=2.02$; POD 6: $2.74+0.85 \times(-1)=1.89$; POD $7: 2.74+1 \times(-1)=1.76$.
These predicted pain scores over time were close to the mean values directly observed from the data. The RMSEA and CFI values of the basic model were 0.07 and 0.95 , respectively. Figure 1 illustrates the basic model with intercept and slope parameters.

The results of the single predictor latent curve model are presented as online supplemental table 1. Among them, only preoperative use of parecoxib and IVPCA had significant effects on the intercept parameter of pain scores over time. With respect to the slope parameter, preoperative and postoperative use of parecoxib and PCEA were identified as significant predictors.

Table 2 illustrates the result of multiple predictor in the latent curve analysis after model selection. Five independent predictors were associated with the intercept parameter of maximal pain scores over time. Woman, postoperative use of parecoxib and body weight shows positive association, but preoperative use of parecoxib and PCEA reveals negative connections on the intercept parameter. In contrast, only age and PCEA was significantly associated with the slope parameter of the maximal pain score over time and both had positive correlations on it.

Based on the above results, the estimated maximal pain score during the first postoperative week can be estimated with the prediction model described in table 3 . For examples, for a 50-year-old female patient with body weight of $60 \mathrm{~kg}$, preoperative use of parecoxib and PCEA, the estimated maximal pain score on the POD 3 is equal to $1.91\left[2.25+0.17 \times{ }^{\prime} 1\right.$ ' (female) $-0.33 \times{ }^{\prime} 1$ ' (PCEA) $+0.24 \times{ }^{\circ} 0$ ' (postoperative use of parecoxib)-0.61 $\times^{\prime} 1$ ' (preoperative use of parecoxib) $+0.01 \times{ }^{\prime} 60$ ' (body weight $)+0.21 \times(-$ $1.79+0.01 \times 550$ ' age $+0.48 \times{ }^{\prime} 1$ ' (PCEA) $]$. The RMSEA and CFI values of the final model were 0.05 and 0.96 , respectively, and the graphic presentation of the final model is illustrated as figure 2.

The results of hurdle Poisson regression analysis of rescue morphine use are presented in table 4. Ageing and postoperative use of parecoxib were significantly associated with increased probability of rescue morphine use but administration of PCEA and IVPCA decreased it. Preoperative parecoxib use was not related to the probability of rescue morphine administration ( $\mathrm{OR}=1.57 ; 95 \%$ CI 0.77 to 3.22 ) but significantly associated with reduced frequency of rescue morphine use among the users of rescue morphine $(\mathrm{HR}=0.4 ; 95 \% \mathrm{CI} 0.25$ to 0.65$)$. Other factors included in the analysis were not related to the frequency of rescue morphine use.

\section{DISCUSSION}

In this study, we applied latent curve analysis to model the longitudinal data of in-hospital postoperative pain scores after thoracic surgeries and found pre-emptive analgesia with parecoxib reduced the baseline level but not the decreasing rate of pain scores over time. Besides, we established a clinical prediction model incorporating patient demographics and analgesic modalities for the change 
Table 1 Comparisons of patient characteristics and postoperative pain scores between patients using preoperative parecoxib and their counterparts

\begin{tabular}{|c|c|c|c|}
\hline & Preoperative parecoxib & Nil & \\
\hline & $(\mathrm{N}=196)$ & $(\mathrm{N}=319)$ & $P$ value \\
\hline Age, years & $59.0 \pm 13.5$ & $61.1 \pm 12.2$ & 0.063 \\
\hline Sex, female & $110(56.1 \%)$ & $192(60.2 \%)$ & 0.363 \\
\hline Body height, $\mathrm{cm}$ & $160.9 \pm 8.3$ & $160.8 \pm 8.8$ & 0.826 \\
\hline Body weight, kg & $62.1 \pm 12.2$ & $62.8 \pm 12.1$ & 0.533 \\
\hline BMI, $\mathrm{kg} \mathrm{m}^{-2}$ & $23.9 \pm 4.0$ & $24.2 \pm 3.7$ & 0.352 \\
\hline ASA class $\geq 3$ & $35(17.9 \%)$ & $61(19.1 \%)$ & 0.720 \\
\hline Diabetes & $13(6.6 \%)$ & $46(14.4 \%)$ & 0.007 \\
\hline Type of tumour & & & 0.970 \\
\hline Benign lesion & $56(28.6 \%)$ & $94(29.5 \%)$ & \\
\hline Adenocarcinoma & $117(59.7 \%)$ & $187(58.6 \%)$ & \\
\hline Other malignancy & $23(11.7 \%)$ & $38(11.9 \%)$ & \\
\hline Clinical stage of disease & & & 0.130 \\
\hline Benign or stage 0 & $104(53.1 \%)$ & $155(48.6 \%)$ & \\
\hline Stage 1-2 & $82(41.8 \%)$ & $137(42.9 \%)$ & \\
\hline Stage 3-4 & $10(5.1 \%)$ & $27(8.5 \%)$ & \\
\hline \multicolumn{4}{|l|}{ Analgesic modalities } \\
\hline Postoperative use of parecoxib & $181(92.3 \%)$ & $29(9.1 \%)$ & $<0.001$ \\
\hline PCEA & $15(7.7 \%)$ & $188(58.9 \%)$ & $<0.001$ \\
\hline IVPCA & $4(2.0 \%)$ & $76(23.8 \%)$ & $<0.001$ \\
\hline Anaesthesia time, min & $195(150-248)$ & $210(165-255)$ & 0.070 \\
\hline Maximal NRS pain scores & & & $<0.001$ \\
\hline POD 1 & $2.6 \pm 1.3$ & $2.7 \pm 1.3$ & 0.291 \\
\hline POD 2 & $2.7 \pm 1.3$ & $2.9 \pm 1.3$ & 0.041 \\
\hline POD 3 & $2.3 \pm 1.1$ & $2.5 \pm 1.1$ & 0.010 \\
\hline POD 4 & $1.9 \pm 0.9$ & $2.3 \pm 1.0$ & $<0.001$ \\
\hline POD 5 & $1.7 \pm 0.8$ & $2.3 \pm 1.2$ & $<0.001$ \\
\hline POD 6 & $1.7 \pm 1.0$ & $2.1 \pm 1.2$ & 0.001 \\
\hline POD 7 & $1.6 \pm 1.1$ & $2.0 \pm 1.3$ & 0.011 \\
\hline Use of rescue morphine & $139(70.9 \%)$ & $84(26.3 \%)$ & $<0.001$ \\
\hline Frequency of rescue morphine ${ }^{\star}$ & $1(1-1)$ & $1(1-2)$ & 0.092 \\
\hline Length of hospital stay, day & $5(4-7)$ & $7(6-8)$ & $<0.001$ \\
\hline
\end{tabular}

Values were mean $\pm S D$ deviation, counts (per cent) or median (IQR).

*Among those who used rescue morphine.

ASA, American Society of Anesthesiologists; BMI, body mass index; IVPCA, intravenous patient-controlled analgesia; NRS, numeric rating scale; PCEA, patient-controlled epidural analgesia; POD, postoperative day.

of in-hospital pain trajectories, which provided valuable implications for perioperative pain management.

A number of randomised controlled trials have demonstrated the efficacy of intravenous administration of parecoxib as pre-emptive analgesia against placebo. ${ }^{4-79}$ Pre-emptive analgesia with parecoxib significantly reduced postoperative pain scores and postoperative opioid consumption among patients undergoing thoracic surgery, ${ }^{45}$ gynaecological abdominal surgery ${ }^{67}$ and spine surgery. ${ }^{9}$ Our results were in line with the preceding studies. Furthermore, our study contributed to revealing the complex relationship between dynamic changes of postoperative subjective pain scores over time, various types of analgesic modalities and patient characteristics in the clinical setting of video-assisted thoracoscopic surgery. Our analysis demonstrated the independent effect of preemptive analgesia with parecoxib mainly exerted on the baseline value (intercept) rather than the decreasing rate (slope) of postoperative pain scores. In other words, preemptive analgesia with parecoxib significantly reduced 


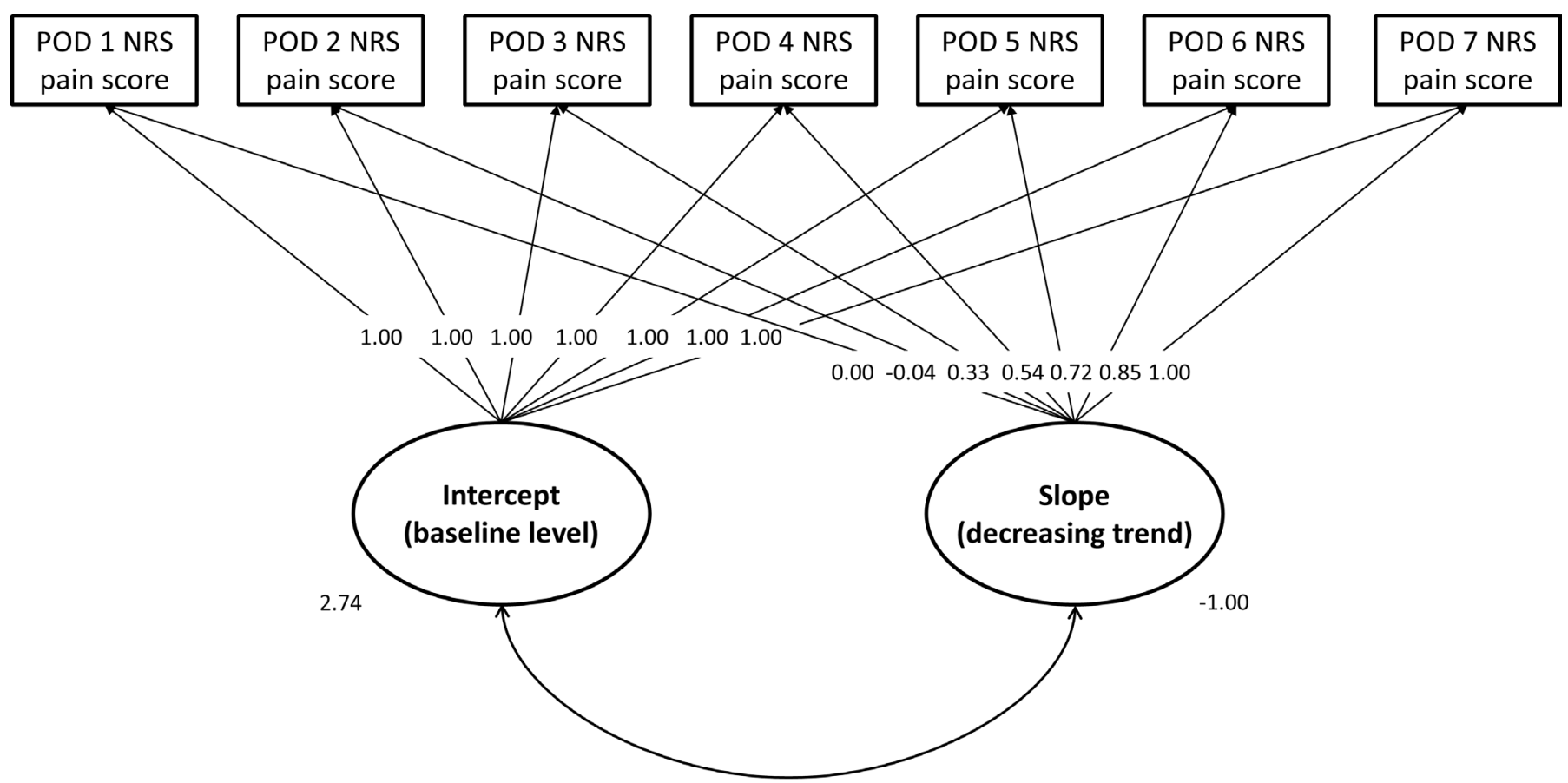

Chi-square $=60.354, \mathrm{CFI}=0.945, \mathrm{RMSEA}=0.068$

Figure 1 Basic model of latent curve analysis. CFI, comparative fit index; NRS, numeric rating scale; POD, postoperative day; RMSEA, root mean square error of approximation.

maximal pain scores over time by universally lowering the baseline pain level during the postoperative period, without flattening or steepening the decreasing trend of pain scores. A possible explanation of this finding is that the effect of pre-emptive analgesia with parecoxib mainly comes from blocking the release of cytokines which mediate inflammation, including interleukin 6 (IL-6) and IL-8, and consecutively terminating the cascade of peripheral sensitisation to eventual central sensitisation. ${ }^{18}$ To be noticed, although postoperative administration of parecoxib was associated with increasing baseline level of postoperative pain, this did not imply that administration

Table 2 Results of multiple predictor latent curve models after backward model selection

\begin{tabular}{|c|c|c|c|}
\hline & Estimate & SE & $\begin{array}{l}P \\
\text { value }\end{array}$ \\
\hline \multicolumn{4}{|l|}{ Intercept } \\
\hline Sex (female vs male) & 0.167 & 0.079 & 0.035 \\
\hline PCEA & -0.331 & 0.088 & $<0.001$ \\
\hline $\begin{array}{l}\text { Postoperative use of } \\
\text { parecoxib }\end{array}$ & 0.241 & 0.122 & 0.048 \\
\hline Preoperative use of parecoxib & -0.606 & 0.125 & $<0.001$ \\
\hline Body weight & 0.009 & 0.003 & 0.005 \\
\hline \multicolumn{4}{|l|}{ Slope } \\
\hline Age & 0.010 & 0.004 & 0.014 \\
\hline PCEA & 0.477 & 0.121 & $<0.001$ \\
\hline
\end{tabular}

PCEA, patient-controlled epidural analgesia. of parecoxib after surgery made postoperative pain worse but the otherwise: patients suffered from more severe postoperative pain tended to be administered parecoxib as an adjunct for pain control. Additionally, our analysis unveiled that PCEA had a significant effect on both baseline level and decreasing rate of pain scores over time. Taken together with these results, patients receiving preemptive parecoxib may have lower baseline level of postoperative pain, but still, need additional analgesics for faster pain resolution or management of breakthrough pain in a clinical scene. According to our results, PCEA may remain an ideal option of pain control in the setting of in-hospital pain following thoracic surgery if there is no contraindication for epidural placement. This proposal was supported by some experts. ${ }^{19}$ Our results showed IVPCA had no significant effect on neither baseline level nor the decreasing rate of pain scores over time. This finding was supported by another study. ${ }^{20}$

The sex difference in the postoperative pain level and analgesic demand remained controversial in the previous literature. ${ }^{2-23}$ Some studies suggested that woman tended to report greater postoperative pain scores after several types of surgery, including thoracic surgery. ${ }^{24} 25$ The present study also found a significant sex difference in postoperative pain scores, suggesting woman as a risk factor for higher baseline pain scores. On the other hand, we found no significant sex difference in the decreasing trend of pain score over time. This result was consistent with our prior study which also applied latent curve analysis, revealing that women undergoing laparoscopic colorectal surgery had similar decreasing trend of 
Table 3 The prediction model of the estimated pain score during the first operative week

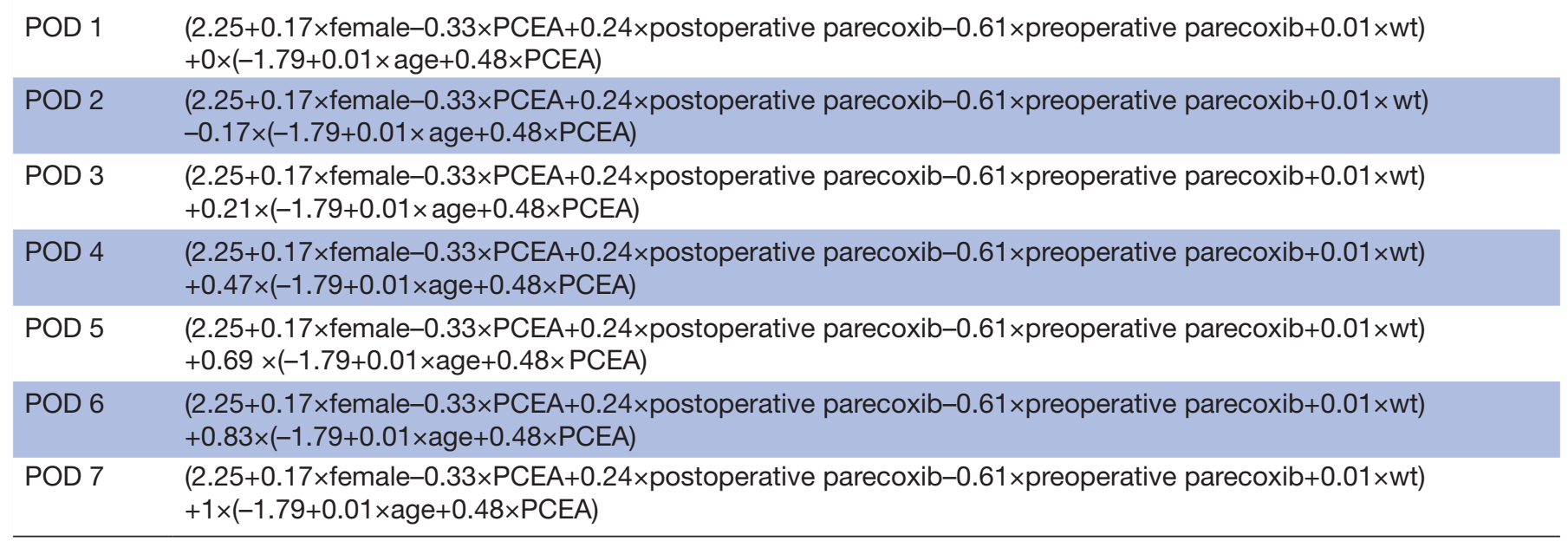

PCEA, patient-controlled epidural analgesia; POD, postoperative day; wt, weight in kilograms.

postoperative patient-controlled morphine consumption to male patients. ${ }^{10}$

Gerbershagen and colleagues reported that younger age was associated with higher postoperative pain intensity on the first POD, independently of types of surgery and presence of preoperative chronic pain. ${ }^{24}$ Our analysis further discovered that the maximal pain score decreased faster in older patients, contrasting with some studies. ${ }^{26}$ The discrepant finding may be explained by the difference in patient characteristics, surgical types, and analgesic management.

Prior studies suggested body weight was not related to pain intensity in patients undergoing cardiac surgery nor predictive of analgesic demands in patients following abdominal surgery. ${ }^{1027}$ Our study found that higher body weight tended to increase the baseline pain intensity. However, the effect size was relatively small, and the clinical significance of this finding should be interpreted carefully.

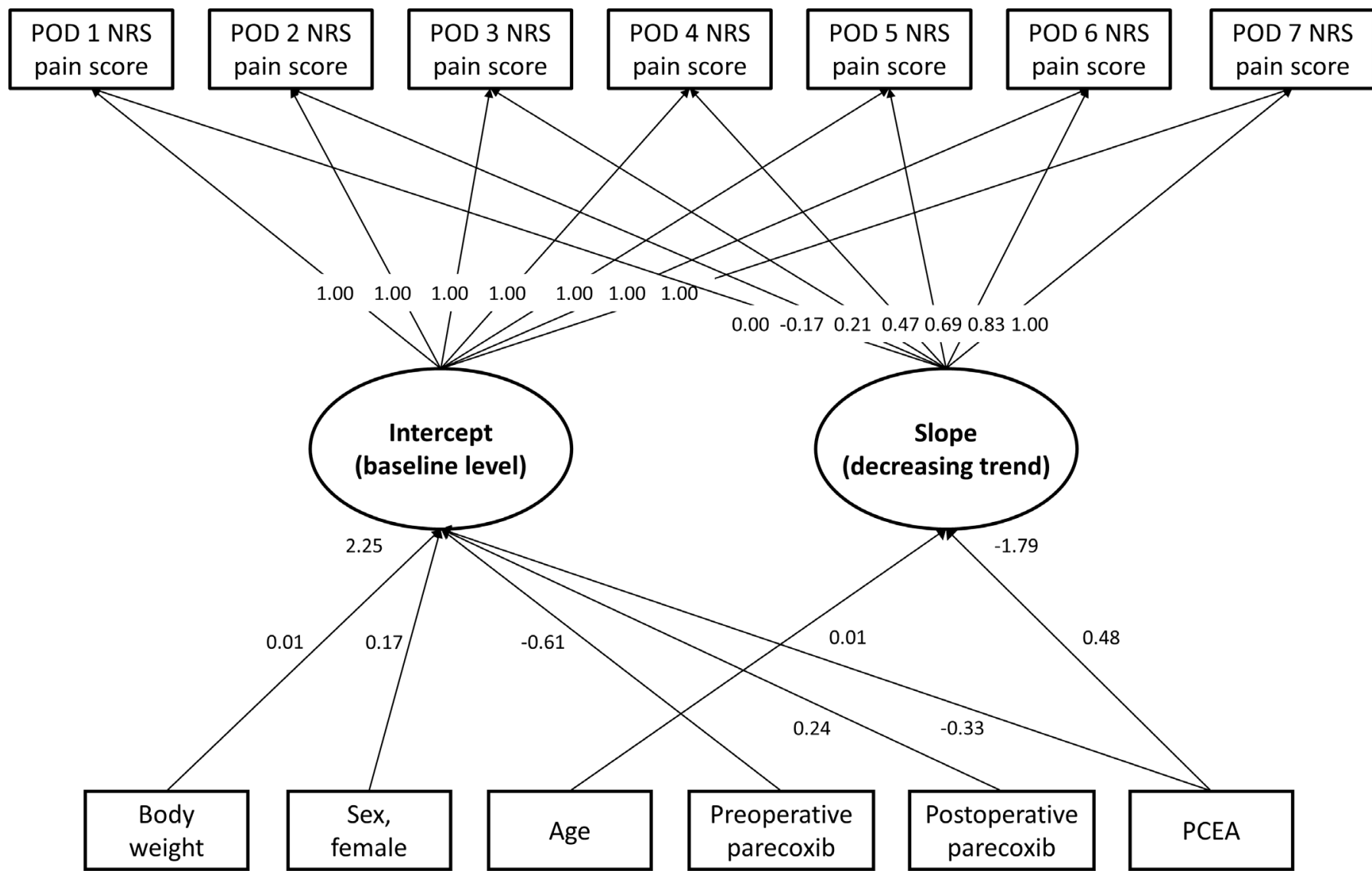

Figure 2 Final model of latent curve analysis. NRS, numeric rating scale; POD, postoperative day. 
Table 4 Hurdle Poisson regression analysis of the probability and frequency of rescue morphine use during the first three postoperative days

\begin{tabular}{|c|c|c|c|c|c|c|}
\hline & \multicolumn{3}{|c|}{ Probability of rescue morphine use } & \multicolumn{3}{|c|}{ Frequency of rescue morphine use } \\
\hline & OR & $95 \% \mathrm{Cl}$ & $P$ value & HR & $95 \% \mathrm{Cl}$ & $\begin{array}{l}P \\
\text { value }\end{array}$ \\
\hline Age & 1.03 & 1.01 to 1.05 & 0.001 & 0.99 & 0.98 to 1.01 & 0.331 \\
\hline Sex (female vs male) & 1.04 & 0.64 to 1.70 & 0.865 & 0.97 & 0.66 to 1.41 & 0.856 \\
\hline Body weight & 1.00 & 0.98 to 1.02 & 0.918 & 1.00 & 0.98 to 1.01 & 0.891 \\
\hline Preoperative use of parecoxib & 1.57 & 0.77 to 3.22 & 0.215 & 0.40 & 0.25 to 0.65 & $<0.001$ \\
\hline Postoperative use of parecoxib & 2.61 & 1.33 to 5.15 & 0.006 & 1.36 & 0.84 to 2.21 & 0.209 \\
\hline PCEA & 0.36 & 0.20 to 0.63 & $<0.001$ & 0.76 & 0.48 to 1.23 & 0.263 \\
\hline IVPCA & 0.20 & 0.09 to 0.43 & $<0.001$ & 0.51 & 0.20 to 1.28 & 0.151 \\
\hline
\end{tabular}

IVPCA, intravenous patient-controlled analgesia; PCEA, patient-controlled epidural analgesia.

There are some limitations in this study. First, it is difficult to assess potential confounding effects from unmeasured variables, such as preoperative pain and psychosocial factors. ${ }^{28}$ Although our final model acceptably fit the data, further investigation is required to clarify the relationship between these factors and postoperative pain level and to recognise the efficacy of parecoxib as pre-emptive analgesia in specific subgroups of surgical patients. Second, only patients undergoing thoracic surgery were included in this study. The effect of preemptive analgesia with parecoxib on the postoperative pain score in other types of surgery awaits further investigation. However, it was also a strength that the heterogeneity of the studied subjects was thereby decreased, ensuring the analytical models free of potential surgeryrelated cofounders. Third, we applied the numerical rating score as an indicator of pain intensity, instead of postoperative analgesics demand. The numerical rating score is a direct but relatively subjective indicator in the measurement of pain intensity. The validity and reliability of self-report pain rating scale in Asian population has been verified by prior studies, ${ }^{29}$ but biases may exist in some certain subpopulations. ${ }^{30}$ Besides, this subjective parameter may also be affected by social status, educational background, cognitive ability of the patients and so on. Fourth, all of the pain scores were assessed at rest by nurses in charge and how postoperative pain trajectories varied during coughing or moving were not further evaluated due to data unavailability.

\section{CONCLUSION}

In conclusion, our analysis using latent curve models demonstrated that age, sex, body weight, epidural analgesia, preoperative and postoperative parecoxib were explanatory factors of postoperative pain variability in patients undergoing video-assisted thoracoscopic surgery. Pre-emptive analgesia with parecoxib reduced baseline pain scores but did not affect their decreasing trend over time.
Author affiliations

${ }^{1}$ Department of Neurology, Neurological Institute, Taipei Veterans General Hospital, Taipei, Taiwan

${ }^{2}$ School of Medicine, National Yang-Ming University, Taipei, Taiwan

${ }^{3}$ Department of Anesthesiology, Taipei Veterans General Hospital, Taipei, Taiwan

${ }^{4}$ Department of Anesthesiology, Shuang Ho Hospital, Taipei Medical University, New Taipei City, Taiwan

${ }^{5}$ Department of Anesthesiology, School of Medicine, College of Medicine, Taipei Medical University, Taipei, Taiwan

Contributors Y-HL contributed to manuscript drafting and data collection. Y-HT contributed to manuscript drafting and revision. H-LW, W-LF and M-YT helped revise the manuscript. K-YC contributed to statistical analysis, manuscript revision and gave final approval of the version to be published and agreed to be accountable for ensuring the accuracy or integrity of the work. All authors read and approved the final manuscript.

Funding This work was funded by the grants from Taipei Veterans General Hospital, Taipei, Taiwan (V106C-048), Ministry of Science and Technology, Taipei, Taiwan (MOST 107-2511-H-075-001, MOST108-2511-H-075-001), Yen Tjing Ling Medical Foundation (Cl-109-29) and Anesthesiology Research and Development Foundation, Taipei, Taiwan (ARDF10701).

Competing interests None declared.

Patient consent for publication Not required.

Ethics approval The study was approved by the Institutional Review Board of Taipei Veterans General Hospital, Taipei, Taiwan (IRB-TPEVGH No. 2015-11-010CC). Written informed consent was waived and all the study materials were anonymised and deidentified before processing.

Provenance and peer review Not commissioned; externally peer reviewed.

Data availability statement № data are available. Data are available upon reasonable request but the approval of institutional review board will be necessary. Please contact the corresponding author for detail.

Supplemental material This content has been supplied by the author(s). It has not been vetted by BMJ Publishing Group Limited (BMJ) and may not have been peer-reviewed. Any opinions or recommendations discussed are solely those of the author(s) and are not endorsed by BMJ. BMJ disclaims all liability and responsibility arising from any reliance placed on the content. Where the content includes any translated material, BMJ does not warrant the accuracy and reliability of the translations (including but not limited to local regulations, clinical guidelines, terminology, drug names and drug dosages), and is not responsible for any error and/or omissions arising from translation and adaptation or otherwise.

Open access This is an open access article distributed in accordance with the Creative Commons Attribution Non Commercial (CC BY-NC 4.0) license, which permits others to distribute, remix, adapt, build upon this work non-commercially, and license their derivative works on different terms, provided the original work is 
properly cited, appropriate credit is given, any changes made indicated, and the use is non-commercial. See: http://creativecommons.org/licenses/by-nc/4.0/.

\section{ORCID iD}

Kuang-Yi Chang http://orcid.org/0000-0002-9062-5519

\section{REFERENCES}

1 Kissin I. Preemptive analgesia. Anesthesiology 2000;93:1138-43.

2 Kelly DJ, Ahmad M, Brull SJ. Preemptive analgesia I: physiological pathways and pharmacological modalities. Can $J$ Anaesth 2001;48:1000-10.

3 Nir R-R, Nahman-Averbuch H, Moont R, et al. Preoperative preemptive drug administration for acute postoperative pain: a systematic review and meta-analysis. Eur J Pain 2016;20:1025-43.

4 Pipanmekaporn T, Punjasawadwong Y, Charuluxananan S, et al. The effectiveness of intravenous parecoxib on the incidence of ipsilateral shoulder pain after thoracotomy: a randomized, double-blind, placebo-controlled trial. J Cardiothorac Vasc Anesth 2018;32:302-8.

5 Lu J, Liu Z, Xia K, et al. Effect of preemptive analgesia with parecoxib sodium in patients undergoing radical resection of lung cancer. Int $J$ Clin Exp Med 2015;8:14115-8.

6 Zhang $\mathrm{H}$, Liu $\mathrm{X}$, Jiang $\mathrm{H}$, et al. Parecoxib increases muscle pain threshold and relieves shoulder pain after gynecologic laparoscopy: a randomized controlled trial. J Pain Res 2016;9:653-60.

7 Liu W-F, Shu H-H, Zhao G-D, et al. Effect of parecoxib as an adjunct to patient-controlled epidural analgesia after abdominal hysterectomy: a multicenter, randomized, placebo-controlled trial. PLoS One 2016;11:e0162589.

8 Peng H-M, Wang L-C, Wang W, et al. Preemptive analgesia with parecoxib in total hip arthroplasty: a randomized, double-blind, placebo-controlled trial. Pain Physician 2018;21:483-8.

9 Siribumrungwong K, Cheewakidakarn J, Tangtrakulwanich B, et al. Comparing parecoxib and ketorolac as preemptive analgesia in patients undergoing posterior lumbar spinal fusion: a prospective randomized double-blinded placebo-controlled trial. BMC Musculoskelet Disord 2015;16:59.

10 Chao P-W, Lin S-P, Tsou M-Y, et al. Assessing the impact of renal function on trajectory of intravenous patient-controlled analgesic demands over time after open and laparoscopic colorectal surgery using latent curve analysis. Clin J Pain 2016;32:695-701.

11 Lo P-H, Tsou M-Y, Chang K-Y. Modeling the trajectory of analgesic demand over time after total knee arthroplasty using the latent curve analysis. Clin J Pain 2015;31:776-81.

12 Lin S-P, Chang K-Y, Chen $\mathrm{H}-\mathrm{H}$, et al. Predicting procedure-specific morphine consumption of intravenous patient-controlled analgesia with Random-effect model approach. Clin J Pain 2019;35:43-9.
13 Bollen KA, Curran PJ. Latent curve models: a structural equation perspective. Hoboken: John Wiley \& Sons, 2006.

14 Preacher KJ, Wichman AL, MacCallum RC. Latent growth curve modeling. New York: Sage, 2008.

15 Byrne BM. Structural equation modeling with MPLUS: basic concepts, applications, and programming. Abingdon-on-Thames: Routledge, 2013.

16 Kline RB. Principles and practice of structural equation modeling. New York: Guilford publications, 2015.

17 Mullahy J. Specification and testing of some modified count data models. J Econom 1986;33:341-65.

18 Pandazi A, Kapota E, Matsota P, et al. Preincisional versus postincisional administration of parecoxib in colorectal surgery: effect on postoperative pain control and cytokine response. A randomized clinical trial. World J Surg 2010;34:2463-9.

19 Mesbah A, Yeung J, Gao F. Pain after thoracotomy. BJA Educ 2016;16:1-7.

20 Cheung CW, Ying CLA, Lee LHY, et al. An audit of postoperative intravenous patient-controlled analgesia with morphine: evolution over the last decade. Eur J Pain 2009;13:464-71.

21 Aubrun F, Salvi N, Coriat P, et al. Sex- and age-related differences in morphine requirements for postoperative pain relief. Anesthesiology 2005;103:156-60.

22 Ip HYV, Abrishami A, Peng PWH, et al. Predictors of postoperative pain and analgesic consumption: a qualitative systematic review. Anesthesiology 2009;111:657-77.

23 Schnabel A, Poepping DM, Gerss J, et al. Sex-Related differences of patient-controlled epidural analgesia for postoperative pain. Pain 2012:153:238-44.

24 Gerbershagen HJ, Pogatzki-Zahn E, Aduckathil S, et al. Procedurespecific risk factor analysis for the development of severe postoperative pain. Anesthesiology 2014;120:1237-45.

25 Tighe PJ, Riley JL, Fillingim RB. Sex differences in the incidence of severe pain events following surgery: a review of 333,000 pain scores. Pain Med 2014;15:1390-404.

26 Tighe PJ, Le-Wendling LT, Patel A, et al. Clinically derived early postoperative pain trajectories differ by age, sex, and type of surgery. Pain 2015;156:609-17.

27 Mueller XM, Tinguely F, Tevaearai HT, et al. Pain location, distribution, and intensity after cardiac surgery. Chest 2000;118:391-6.

28 Katz J, Poleshuck EL, Andrus $\mathrm{CH}$, et al. Risk factors for acute pain and its persistence following breast cancer surgery. Pain 2005;119:16-25.

29 Li L, Liu X, Herr K. Postoperative pain intensity assessment: a comparison of four scales in Chinese adults. Pain Med 2007;8:223-34.

30 Gagliese L, Katz J. Age differences in postoperative pain are scale dependent: a comparison of measures of pain intensity and quality in younger and older surgical patients. Pain 2003;103:11-20. 